\title{
SOBRETRABAJO EN TIEMPOS DEL COVID-19: DESVELANDO LAS JORNADAS DE MUJERES EN EL CONFINAMIENTO
}

\author{
OVERWORK IN COVID-19 TIMES: UNVEILING WOMEN'S JOURNEYS IN \\ CONFINEMENT
}

\author{
José Luis, CISNEROS ${ }^{1}$ \\ Dora del Carmen, YAUTENTZI DÍAZ ${ }^{2}$
}

\begin{tabular}{|lll|}
\hline Recibido & $:$ & 21.11 .2020 \\
Aceptado & $:$ & 21.12 .2020 \\
Publicado & $:$ & 12.02 .2021 \\
\hline
\end{tabular}

\begin{abstract}
RESUMEN: Este trabajo es el resultado de una investigación que tiene como propósito ofrecer al lector una perspectiva sobre las jornadas de trabajo experimentadas por las mujeres durante el tiempo de pandemia por el COVID-19, nuestra finalidad es acercarnos a reflexionar sobre la violencia estructural, y sus implicaciones culturales y económicas. Particularmente nos interesa subrayar los efectos experimentados en la jornada laboral que, con motivo del confinamiento, se ha exacerbado. Nuestra investigación es de corte etnográfico en tanto que nos interesa dar cuenta de la experiencia que viven las mujeres, solo así, consideramos que se puede voz a ellas como actores principales, que narran su experiencia y expresar su percepción acerca de las implicaciones que ha traído este tiempo de pandemia en su condición de mujer. Cuantitativamente nos apoyamos en la aplicación de encuestas mediante las redes sociales. Nuestros principales hallazgos evidencian una condición de vulnerabilidad cansancio y devastación, no solo en su condición de mujer, sino en su relación de pareja y con su familia. Sin embargo, a pesar de haber perdido su empleo formal, muchas mujeres han buscado estrategias de subsistencia para sostener a sus familias.
\end{abstract}

Palabras clave: Covid-19, violencia estructural, jornada, trabajo, mujeres.

\begin{abstract}
This work is the result of an investigation that aims to offer the reader a perspective on the work days that women have experienced during the time of the COVID-19 pandemic, our purpose is to get closer to reflecting on structural violence, and its effects on culture, economy. We are particularly interested in highlighting the effects experienced in the working day which, due to confinement, has been exacerbated. This research is of an ethnographic nature insofar as we are interested in giving an account of the experience that women live, only in this way do we consider that we can give a voice to them as main social actors, by narrating their experience and expressing perception about the implications that it has brought this time of pandemic as a woman. Quantitatively, we rely on the application of surveys through social networks. Our main findings show a condition of vulnerability, fatigue and devastation, not only in her condition as a woman, but also in her relationship with her partner and with her family. However, despite having lost their formal employment, many women have sought livelihood strategies to support their families.
\end{abstract}

Keywords: Covid-19, structural violence, working day, work, women.

\footnotetext{
${ }^{1}$ Doctor en Filosofía, Universidad Autónoma Metropolitana-Unidad Xochimilco, Ciudad de México - México. Email: cisneros.joseluis@gmail.com. ORCID: https://orcid.org/0000-0003-3957-3802

${ }^{2}$ Doctora en Estudios Territoriales. Universidad Autónoma de Tlaxcala-Facultad de Ciencias de la Investigación. TlaxcalaMéxico. Email: dorisyautentzi14@gmail.com. ORCID: https://orcid.org/0000-0002-1662-6644
} 


\section{Journal of the Academy $|10|$}

\section{INTRODUCCIÓN}

Hemos vuelto a experimentar el confinamiento después de once años de haber experimentado 25 días de miedo por la contingencia sanitaria del H1N1, hoy como resultado de un nuevo virus, debemos volver a experimentar una difusa y compleja realidad, y tras diez meses de permanecer en casa, nuestras economías se encuentran a la espera de lo que podría ser un futuro complicado en términos no sólo de crecimiento, sino de depresión y recesión económica. Aunada a esta condición, el clima social marcado por las profundad desigualdades, la marginación y la falta de un futuro promisorio, se suman al clima de niños agotados y fastidiados por el encierro en casa, jóvenes desesperados, aburridos y agobiados por la monotonía del encierro, y la economía de los hogares devastada junto al desánimo de muchas mujeres que viven y experimentan otras formas de violencia, tan brutales como la violencia física (Cisneros, 2010).

Viven a diario emociones como ira, enojo, rabia, furor y la desesperación para enfrentarse a resolver los problemas cotidianos, de alimentación, pago de servicios y salud entre los más fundamentales.

Estos meses se han vuelto una eternidad, la pandemia del Covid-19, apareció como una ráfaga que aniquiló sueños y planes de muchas familias. Hoy tras una larga espera, solo quedan fragmentos y la esperanza de volver a la dinámica de hace cinco meses. Muchas familias, mujeres y hombres, quedaron presos de una historia rodeada de desgracia y violencia que se suma a la violencia física que ya se vivía en nuestro país. La pandemia nos hundió en una violencia estructural en la que difícilmente nos recuperamos y que ha hecho frágiles a los que más frágiles eran en nuestra sociedad.

Dentro de estos grupos de sujetos frágiles sin duda están las mujeres, son ellas quienes hoy viven las tensiones del confinamiento y sus implicaciones regidas por la sobrecarga de trabajo, desempleo, y crisis económica. Los recortes al salario y los descansos obligatorios del trabajo sin ingresos son condiciones que se suman a las profundas desigualdades de las que ya eran objeto las mujeres, por ejemplo, la Encuesta sobre el Impacto Económico generado por el Covid-19 (INEGI, 2020) del INEGI, señaló que en el mes de abril de este año, 2020 la tasa de participación de la mujer en el trabajo fue de apenas $36.4 \%$ en comparación a la registrada por los hombres, que fue de un $69.1 \%$, cifras que un mes anterior a la pandemia, registraba $76.5 \%$ 


\section{Journal of the Academy | $11 \mid$}

y $45.4 \%$ respectivamente. Esto implica que la proporción de mujeres que fueron desplazadas del mercado laboral ha sido significativamente mayor, que en el caso de los hombres. Hoy, a cinco meses de iniciado el confinamiento, el $23 \%$ de estas mujeres mantiene un endeble vínculo laboral, algunas con disminución de jornada, otras con recorte de ingresos, y el mayor número de ellas, es decir, el $87.7 \%$ de esto universo, no reciben ningún tipo de apoyo económico. (INEGI, 2020)

Las condiciones enunciadas con anterioridad han sometido a la mujer a un mayor grado de desigualdad y como resultado de esta violencia estructural sumada a los efectos del Covid-19, muchas mujeres hoy han tenido que autoemplearse: vender alimentos, o productos por catálogo, habilitar puestos ambulantes, y en el mejor de los casos tratar de emprender algún pequeño negocio familiar. A este esfuerzo tenemos que sumarle la sobrecarga del trabajo doméstico, que también configura una de las expresiones de violencia que viven muchas de estas mujeres, aunado a sus compromisos como proveedoras, que históricamente y de manera acentuada se ha marcado como una de las condiciones en las que viven estas mujeres en México. Por ejemplo, según datos del Instituto Nacional de Geografía, Estadística e Informática federal, en 49 de cada 100 hogares en nuestro país, una mujer es la jefa de familia; así lo señala la Encuesta Nacional de los Hogares (ENH, 2017), donde se subraya que el 28.5\% de los hogares en México, son comandados por mujeres.

Debemos considerar que conjunto con esta espiral de trabajo se suma también el trabajo de aquellas que aún tienen hijos que asisten a educación preescolar y básica, que tienen que asumir al papel de supervisoras y acompañantes, para apoyar en las tareas escolares. Se trata de un trabajo adicional a las tareas de la limpieza y elaboración de alimentos, son mujeres que además de atender su trabajo, atienden a sus hijos y las actividades domésticas de sus hogares, como lo afirma la Encuesta sobre el Impacto Económico generado por el Covid-19 (2020), cuando señala que del $55.9 \%$ de mujeres que aún mantiene su empleo y trabajan desde sus hogares, además de atender su trabajo, atienden a sus hijos, y las actividades de limpieza y quehacer doméstico, según esta encuesta el 91.9\% de las mujeres realiza trabajo adicional sumado al remunerado, como son las tareas en hogar y cuidado de los padres, hermanos o algún familiar enfermo en comparación a un 78\% de los hombres (INEGI, 2020).

En este sentido, la reflexión que desarrollamos en estas líneas se inscribe en el marco de la reflexión que surge de una investigación más amplia titulada, “El tiempo, en tiempos del 


\section{Journal of the Academy $|12|$}

confinamiento Covid-19. (Se trata de un proyecto de investigación registrado ante el Consejo Académico de la División de Ciencias Sociales y Humanidades de la Universidad Autónoma Metropolitana, Unidad Xochimilco, en el que exploramos la percepción y uso del espacio y el tiempo durante los tres primeros meses de confinamiento.) En este marco, apareció la idea de desarrollar este ensayo con el propósito de documentar de manera más específica el entramado de relaciones que se han creado como resultado de los efectos desencadenados por la pandemia, alrededor del trabajo excesivo que padecen las mujeres en sus hogares, y mostrar como en ese trance, las mujeres han tenido que buscar nuevas estrategias y mecanismos para hacer frente a la violencia estructural que se aparejó a la crisis económica acrecentada por el confinamiento. ¿Cuáles son las estrategias sociales e individuales en que se apoyan las mujeres para resolver sus necesidades cotidianas en la manutención de su familia? ¿El confinamiento ha implicado el uso de mayor tiempo de trabajo en tareas domésticas? ¿El confinamiento y la condición laboral han desencadenado emociones y sentimientos en las mujeres?

Metodológicamente, nos hemos apoyado tanto en datos oficiales extraídos de encuestas difundidas por el Instituto Nacional de Estadística, Geografía e Informática (2020) como en datos de una encuesta propia; de igual manera en información cualitativa, obtenida por medio de entrevistas directas semiestructuradas, que fueron aplicadas con mujeres que accedieron a ser grabadas. Es pertinente subrayar que siempre que trabajamos con entrevistas, nuestras investigaciones corren un gran riesgo de subjetividad, en tanto que los datos que obtenemos siempre son reconstruidos desde una experiencia personal. Sin embargo, la recolección de la información tiene un propósito, que es mostrar una visión de la realidad, desde la voz del sujeto en cuestión.

Con relación a la información empírica, esta se recupera de 553 encuestas aplicadas en redes sociales, durante el trascurso de 7 días, del 24 al 30 de abril de 2020; de los instrumentos aplicados, el $65.3 \%$ corresponde a mujeres y el $34.7 \%$ a hombres; con un rango de edad entre 18 y 79 años. Para los fines académicos y metodológicos de este trabajo nos apoyamos exclusivamente en los datos correspondientes a mujeres.

Este trabajo se estructura de dos partes: primero partimos del significado de violencia estructural y después en este sentido ponemos al alcance del lector la idea del sujeto frágil, como aquel sujeto cuyas condiciones de desigualdad y discriminación laboral, social y cultural, no solo es objeto de una violencia física, también en muchas ocasiones de manera inconsciente, 


\section{Journal of the Academy $|13|$}

pasa inadvertido o invisibilizado por la dinámica cultural de nuestra sociedad. Posteriormente, apoyados en nuestro concepto y contextualización del impacto del Covid-19 en México, incorporamos una reflexión de los efectos que ha desencadenado la pandemia en el sobretrabajo de la mujer y sus implicaciones en una gama de emociones y sentimientos. Finalmente se concluye, cómo las mujeres a pesar de que actualmente son destinatarias de una gran cantidad de programas y políticas públicas de apoyo, por motivos de orden cultual siguen siendo receptoras de una violencia combinada.

\section{DESARROLLO}

\section{Violencia estructural en este grupo social}

En México hemos sido testigos de innumerables crisis, generaciones enteras han vivido en la pobreza, como una suerte de maldición, que nos acompaña generación tras generación. Ninguna promesa política desde los años 80 , ha sido certera para sacar al país de la pobreza y la profunda desigualdad, por el contrario, la corrupción, la violencia y la desigualdad se acrecientan sexenio tras sexenio. Las políticas gubernamentales y los programas solo buscan un objetivo, el clientelismo y las vendettas políticas, y no la solución de aquellos problemas que agudizan la marginación y la desigualdad (Hernández, 2006).

Esta condición no solo ha permeado lo económico, social y cultural. En la población, ha propiciado un cambio en el comportamiento social, una suerte de violencia anomia producto de estas evidentes desigualdades que laceran las condiciones básicas de miles de mexicanos e impiden tener acceso a los satisfactores más elementales; salud, vivienda, educación, alimentación y trabajo. Decimos que se trata de una violencia anómica (Cisneros, 2015); en tanto que son condiciones visibles, que no se ocultan y que con el paso del tiempo se han sumado a otros problemas sociales que terminan convirtiéndose en una suerte de acciones de ruptura, presión y desorden cooptado por la agenda de lo político, para capitalizar su fuerza y ganar adeptos mediante programas paliativos de gobierno, que no solucionan de raíz los problemas sociales, y terminan acrecentado los espacios de ilegalidad ante cualquier emergencia a las que nos enfrentamos como país.

Lo anterior, se puede constatar en nuestra historia reciente, bastaría con recordar cualquier fenómeno de orden natural y externo a los cálculos de los gobernantes en turno que se inscriben 


\section{Journal of the Academy $|14|$}

como un mal absoluto que desestabiliza las buenas intenciones de planes y programas de gobierno en el poder; así lo ha mostrado el paso de huracanes, temblores, erupciones volcánicas, enfermedades y epidemias, que termina evidenciando las profundas debilidades de la política en turno y los grandes pendientes de agendas sociales que aún siguen sin resolverse.

Esta condición, es el resultado de una violencia estructural, que tiende a mostrar tras cada fenómeno inesperado, las debilidades orgánicas e inherentes a las formas de gobernar. Aquí la violencia estructural la entendemos como aquellos efectos objetivos que, a pesar de ser diseñados con un carácter de beneficio colectivo, asociados al Estado, las formas de gobierno y las políticas y planes diseñados para buscar el desarrollo integral del bienestar individual y colectivo de la sociedad, logran efectos contrarios en los ciudadanos.

El ejemplo más evidente, es que los mexicanos y mujeres en particular están viviendo un desajuste estructural de sobrecarga de trabajo como resultado de la pandemia Covid-19 y las restricciones domiciliarias que se han impuesto como medida de contención para evitar contagios y crisis de salud más severas. Lo paradójico en muchos países, incluyendo el nuestro, es que la pandemia no solo ha revelado las profundas desigualdades y debilidades en materia de política pública sino que hizo visible la violencia estructural en muchos gobiernos: en México nos recordó la falta de empleos bien remunerados, el gran porcentaje de mexicanos que no tienen acceso a un servicio de salud, miles de mexicanos que no cuentan con servicio de internet y miles de niños que no tienen computadora en casa, que no desayunan y cuyas madres tienen que salir a trabajar para mantener a sus familias y deben encargarlos con alguien o simplemente a la deriva, o bajo cuidado de sus hermanos mayores, aun cuando en muchos casos son también menores de edad. Esta violencia nos muestra el tamaño del empleo informal y las condiciones de ilegalidad en la que muchas mujeres son contratadas, con salarios mal remunerados y sin ninguna garantía social.

Por ejemplo, según datos del Instituto Mexicano del Seguro Social (IMSS) la participación de la mujer en trabajos formales ha aumentado 38\%, lo que implica 1.4 millones de mujeres en el mercado laboral, con un salario promedio de 346 por día, en comparación a los hombres que perciben 378 peros, es decir, una diferencia de 32 pesos menos. Además, se indica que, en diciembre de 2019, el sector de la industria, el comercio y servicios, era ocupado por un $70.8 \%$ de hombres y mujeres, con empleo formal, pero las mujeres concentraban el mayor porcentaje (74.5\%) y los hombres el 68.4\%. Sin embargo, aproximadamente el 74\% de las mujeres con 


\section{Journal of the Academy | 15 |}

actividad laboral en alguno de estos sectores, ganaban menos de tres UMA's (unidad de medida y actualización, es una referencia económica para sustituir el esquema de veces de salario mínimo) (Monroy, 2020a).

De esta manera, el Covid-19, ha venido para recordar que, en nuestro país continúan existiendo algunas particularidades que convierte a muchos mexicanos en sujetos frágiles, hablamos de personas cuyas condiciones de vida, se encuentra a la deriva o en riesgo social, por decirlo así. Son sujetos que como resultado de las condiciones políticas y económicas que se viven en México, se ven afectados en sus condiciones psicológicas, emocionales, sociales y culturales. Los efectos son evidentes en muchos de estos sujetos, las tensiones a las que son sometidos por las presiones sociales y económicas, los somete a una fragilidad que puede expresarse en un extremo, en violencia física, e incluso en el suicidio.

En otros casos, depresión y ruptura de lazos sociales, aislamientos y enfermedades somatizadas, son sujetos que siempre ponen en evidencia su condición de vulnerabilidad, este sería el caso de muchas mujeres que, tras el confinamiento, como resultado de la pandemia, hoy se encuentra en un estado de alta vulnerabilidad; sin empleo, violentadas, con sobrecarga de trabajo, con fatiga emocional, devaluadas y con baja auto estima.

Así, niños, ancianos y mujeres son los ciudadanos más vulnerables en una sociedad como la nuestra. Una sociedad sostenida por un clima de inseguridad y miedo que se extiende ante la presencia de fenómenos adversos, a su propia condición y que se acentúan como resultado no solo de la respuesta omisa y complaciente de las propias autoridades, también se suma a la acción de una violencia directa contra las mujeres, por ejemplo la acciones de violencia contra la mujeres en lo que va de este año 2020, han registrado 280 feminicidios en menores de 17 años, y en los meses de confinamiento, la violencia física en los hogares se incrementó un 66\% y la psicoemocional un 22\%. (Monroy, 2020b). 


\section{Journal of the Academy | $16 \mid$}

\section{Figura 1:}

Tipos de violencias contra la mujer en México.

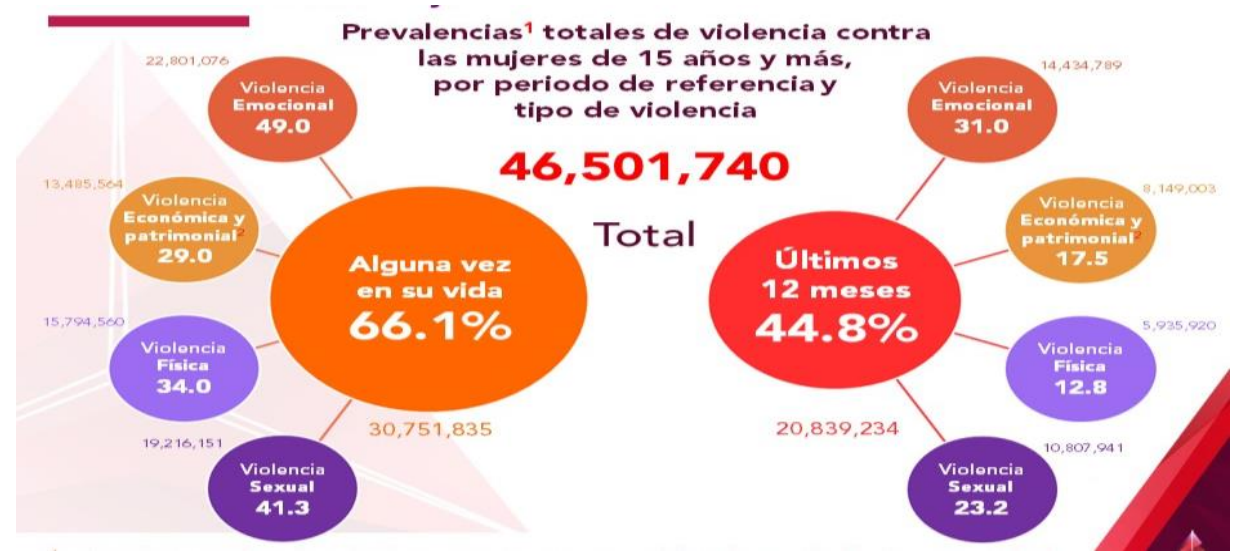

Nota: Violencia contra las mujeres al alza en todo México. Delphos Nudege. UNIT (2020)

La violencia física, cruel y extrema es otra de los grandes temas que se han mostrado como resultado del coronavirus y que pone en evidencia estos sujetos frágiles, así lo demuestran los datos del Secretariado Ejecutivo del Sistema Nacional de Seguridad Pública (SESNSP), que ha registrado en estos meses 320 homicidios de mujeres, es decir 10 casos por día, además de 4 mil 588 denuncias por lesiones dolosas. (Reza, 2020)

El cautiverio no solo asfixia económicamente a la mujer, también la pone en un punto de quiebre y exposición a la violencia de manera conjunta con los hijos y ancianos, así lo muestran los datos reportados por el informe de la Relatora Especial de las Naciones Unidas sobre la violencia contra la mujer, que en cinco meses del confinamiento, se han reportados al Secretariado Ejecutivo del Sistema Nacional de Seguridad Pública (SESNSP) $57.2 \%$ de llamadas por violencia familiar, $19.3 \%$ por violencia de pareja, el $22 \%$ por violencia contra la mujer y $1.4 \%$ por violencia sexual. (INEGI -19,2020)

Como se puede observar, la pandemia no solo afectó a los más débiles y vulnerables, nos ha afectado a todos por igual, sus efectos son muchos como lo hemos estado mencionando, la crisis económica, la pérdida de empleos, y el cierre de negocios que por cierto ha sido el más golpeado y en el que más mujeres laboraban, con un 56\% de su participación. Hablamos también de daños irreparables que no solo se muestran en lo económico, social, cultural y emocional. Sus efectos agudizan la desigualdad y acrecientan la violencia estructural, a la que 


\section{Journal of the Academy | $17 \mid$}

sensatamente el gobierno en turno, ni el siguiente gobierno podrán dar solución por tratarse de un rezago acumulado de deuda social con muchos sectores de la población.

Así, los problemas que hoy nos mostró la pandemia, no son nuevos, son el resultado de varias décadas acumuladas que a mediano plazo no tendrán solución, y que han mostrado las grandes deudas y los cientos de víctimas frágiles que se han acumulado, a las que no se les ha dado respuesta, por ejemplo, el caso de las escuelas, que según el último censo de población, reportó 23,283 establecimientos educativos en los que algunos, no tenían agua entubada, sanitarios deficientes o sin sanitarios, y 20,111 sin luz, una realidad de profundo contrastes, como lo señala Oxfam México (2019) es el 5\% más pobre de mexicano es, corresponde al $2 \%$ más pobre a nivel mundial, y contraste con lo anterior, los más ricos del país se encuentran entre los más ricos a nivel mundial, llegando al percentil 96, la desigualdad en México. (Como se cita en Jaramillo y Vázquez, 2020)

Una desigualdad que se retroalimenta y sostiene con los paliativos de las políticas sociales, que se abonan por las diferencias en los ingresos y la partición de la mujer en la PEA, por ejemplo en México la mujer participa con el 12\% del total nacional de la PEA, sin embargo eso no adquiere importancia hoy, en tiempos del Covid-19, pues las mujeres también se enfrentan como lo hemos mencionado a una mayor carga de trabajo y desigualdad en su casa, por las tareas con los hijos, el cuidado de los familiares, y la atención a un propio empleo remunerado.

\section{El sobretrabajo}

Largas jornadas de trabajo no remunerado, son las que muchas mujeres históricamente han vivido, sumado al trabajo remunerado. En ambos contextos han experimentado desigualdad, vejaciones y maltratos, realizan jornadas casi interminables, se convierten en niñeras, cuidadoras, cocineras, educadoras, prostitutas, secretarias, gestoras, sirvientas, enfermeras y proveedoras (Lagarde, 1993). Son mujeres que organizan su tiempo en un conjunto de actividades cotidianas, donde el ocio y el tiempo de dedicación personal, no está contemplado, su dinámica se concentra en el trabajo doméstico y la atención de los hijos, se trata de un trabajo extraordinario concentrado en actividades primarias por decirlo así, donde se muestran las desigualdades, la pobreza y las tensiones cultuales que las mujeres viven. 


\section{Journal of the Academy $|18|$}

Es un trabajo no reconocido que transfiere valor de la economía productiva a la reproductiva y acentúa la diferenciación entre hombres y mujeres para permanecer en un marcado de trabajo productivo que se esconde en el trabajo doméstico, subestimando su valor en tanto que se realiza en la esfera de lo privado. De esta manera muchas mujeres se ven obligadas a una doble o triple jornada de trabajo con el propósito de amortiguar los efectos de la pérdida de poder adquisitivo, además los sesgos en la condición laboral se hacen más agudos para algunas mujeres, generando angustia y una limitada red de socialización que las impulsa a un vacío social que las hace subestimar su condición y trasferir el limitado tiempo de descanso para realizar tareas complementarias del hogar. (Campillo, 2000) así lo demuestra uno de los testimonios recuperados

"Como mi jornada es matutina, siempre tuve tiempo para venir a comer con mis hijos, mi salida es a las tres de la tarde y vivo a diez minutos de mi centro de trabajo. Cuando mi hijo mayor se fue a la universidad, ... a Guanajuato, se mudó con sus tíos, por esas fechas falleció mi madre que me apoyaba con los hijos y tuve que contratar a una persona para que estuviera con Mariana (su hija menor) y nos hiciera de comer... Yo tenía cierta tranquilidad... llegaba a casa a estar con mi hija y comía con ella; no me preocupaba de la limpieza, una persona me apoyaba y otra señora que guisa dos veces por semana... Cuando se llega este asunto de la pandemia se me vino el mundo encima pues la señora del aseo a veces traía a su nieta y a su hija y la verdad si me dio miedo, de por si el riesgo en mi trabajo y luego más porque por esos días la hija tenía tos y le tuve que pedir que solo viniera una vez por semana y luego la descansé. Lo mismo sucedió con la señora que nos hacía de comer, me dio mucho miedo pues era nuestra comida, así que yo bien optimista pensé que sería cosa de organizar mejor mi tiempo y ahí voy bien valiente: guisaba en la noche, echaba ropa a la lavadora también a esa hora, hacía el quehacer dos veces por semana y le tuve que pedir a mi papá que viniera a estar con Mariana en las mañanas mientras yo iba al trabajo... La tranquilidad me duró unas tres semanas, ... cuando reanudaron clases en línea todo fue de mal en peor. Toda la tarde se me iba en corajes con esta niña y hasta con mi papá. No hacían nada, ni levantar la ropa o secar el baño y llegaba a encontrar los trastes amontonados y la casa asquerosa. Luego me salían con que no les gustaba lo que les hacía de comer y me ponían a hacerles sándwiches o comprábamos pollo, para completar el cuadro luego la niña necesitaba materiales y tenía yo que salir ya toda cansada a la papelería, todo el tiempo estaba ocupada. Cuando llega el periodo vacacional fue peor, porque mi papá y ella despiertan casi a las once de la mañana, cuando yo llego a comer ellos no tienen hambre y yo ya me enfermé de gastritis y colitis nerviosa, aparte no sé si por la careta que uso por lo de la pandemia, ya tengo granos en la cara, en las noches no descanso bien, estoy agotada. La verdad ya quiero que todo esto termine porque los fines de semana se me van en quehaceres que no termino nunca y me siento mal por estar enojada todo el tiempo con mi familia”. (Roldán, 2020)

Se trata de un trabajo oculto, cuya magnitud de tiempo dedicado se vuelve invisible al imponerse y regula la vida de cientos de mujeres, bajo una lógica de reproducción cultural, que sostienen a muchas familias al convertirse en jefas del hogar. Por eso el trabajo no remunerado 


\section{Journal of the Academy $|19|$}

y el tiempo destinado a este adquiere importancia por sus implicaciones en la vida personal, en el uso del tiempo y en las prácticas culturales. Un tiempo que no es valorado ni reconocido, por el contrario, en estos últimos meses de pandemia se ha intensificado y expresado en una doble o triple jornada de trabajo.

\section{Figura 2:}

Distribución de tiempo dedicado a actividades en su hogar

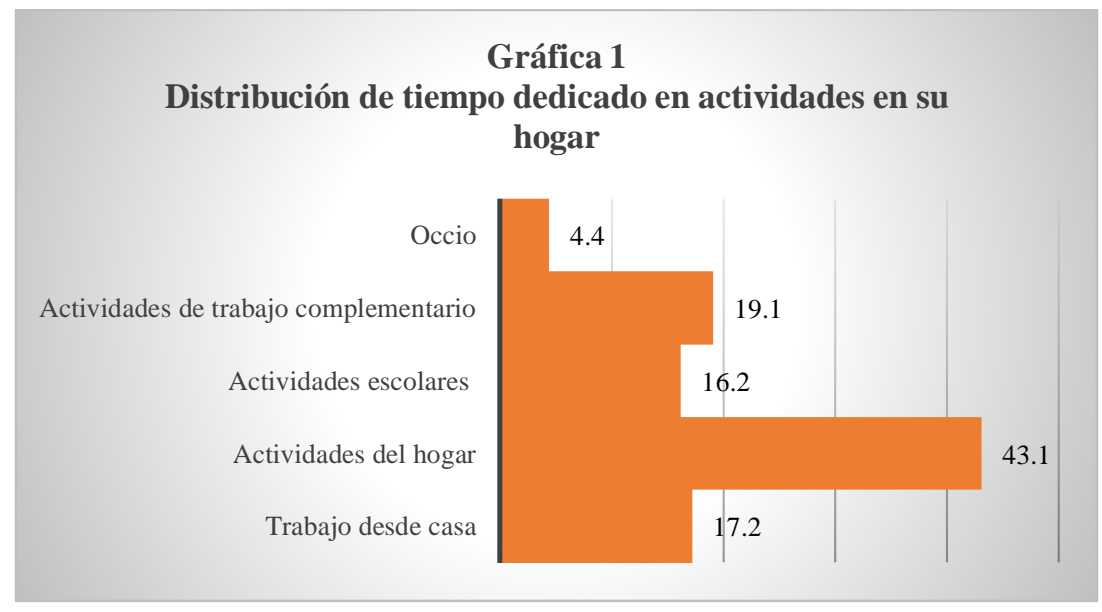

Nota: Elaboración propia con base en encuesta aplicada. 2020. El tiempo en tiempos del confinamiento.

Así, las mujeres en estos seis meses de confinamiento han experimentado un mayor involucramiento y una sobre dimensión de tiempo en actividades y tareas de su hogar, como se aprecia en la gráfica uno, sumado a las actividades del trabajo remunerado. Sin embargo, la prolongación del cautiverio y la parálisis de muchas actividades económicas de nuestra sociedad, han provocado un ensanchamiento en la pobreza y forzado a muchas mujeres a buscar alternativa para el apoyo en sus hogares, tratando de buscar ingresos extras, en una realidad adversa cuya situación económica no favorece a nadie, como lo señalan algunas mujeres.

Los primeros días que todo empezó la gente no vino a la tienda, todos corrieron a la Bodega o Soriana, luego la venta era de cien pesos. Creo que pensaron que ya nadie iba a salir a las calles, ya no me pidieron que fuera a hacer quehaceres a las casas ni me traían ropa de planchar. Todo se vino para abajo, luego cuando las vecinas trabajaban me pedían que les hiciera comida o si tenían pachanga me encargaban que les hiciera tres, cuatro, cinco kilos de arroz, las tortillas, memelitas, que los frijoles charros y así, 


\section{Journal of the Academy $|20|$}

... pero ya no. Mire, igual vendo el Avon y Jafra, y no, nada sale ... ahora, aunque me pidan, no tengo para hacer pedido (suspira)...Como muchas ya vieron que esto de la pandemia ya duró, otra vez empiezan a venir otra vez, pero quieren que fie y luego ya me da pena, porque no sale, ... imagínese o ellos o yo. Ya no he llevado a mi hijo a terapia porque ¿de dónde? La verdad es que luego si me desespero, no me lo va a creer, pero subí mucho de peso y se me hace raro porque no como mucho, no sé qué me pasó. (González, 2020)

Así cada mujer, busca estrategias y dispone de todos los medios posibles para generar algunos ingresos, independientemente de la cantidad de dinero que logre, la mujer dispone de su tiempo para invertirlo como un valor que le apoye en el logro de un bienestar para los integrantes de su familia.

De por sí, mi jornada es pesada pues entro a las siete y media de la mañana, ... en un trabajo que me demanda cuarenta y cinco minutos de traslado. Regreso ya tarde a mi casa, a veces los asuntos, reuniones de gerencias regionales o cuando hay campo no me permiten salir temprano y termino hasta las ocho de la noche, ... voy llegando a las nueve...Con este asunto de la pandemia todo se me complicó; aunque tengo una persona que me apoya en el aseo, yo guiso todas las noches y tengo que abusar de mi hija mayor, ... ella es quien cuida a su hermana. En la noche, llego a hacer la tarea con mi hija y mi problema es que a veces no entendió lo que dijeron en el programa de la televisión... hablaban muy rápido y no le da tiempo copiar... llego y me encuentro los chats de las mamás de la escuela de mi hija saturados de mensajes, entonces a esa hora voy comiendo y contestando o enterándome de los asuntos del salón de mi hija o explicándole los problemas o la tarea. Antes de la pandemia, por lo general llegaba a bañarme, con calma, hoy no puedo, mire... hace mucho que no me corto el cabello y he llegado a dejarme las canas mucho tiempo, pero como estoy frente a clientes tengo que procurar mi arreglo personal. Antes tenía emoción por ir a la estética, pero ahora prefiero comprar de esos tintes de caja... Además, no duermo bien, ando con sueño todo el día y llego rendida la verdad, ... siento que no descanso y he subido de peso, ... creo que es por ansiedad por comer compulsivamente, no tengo horarios, aunque he intentado modificarlos, todo este trabajo, me generan ansiedad”. (Jiménez, 2020)

La realidad es que muchas de estas experiencias, son inéditas para numerosas mujeres, la intensidad de los efectos causados por la pandemia y el confinamiento, han reducido su campo de acción cotidiano, de suerte tal que la presión económica está afectando no solo en la organización de la dinámica de los hogares, también en la condición de salud física y emoción. 


\section{Journal of the Academy $|21|$}

\section{Figura 3:}

Distribución de emociones y sentimientos de mujeres durante la pandemia.

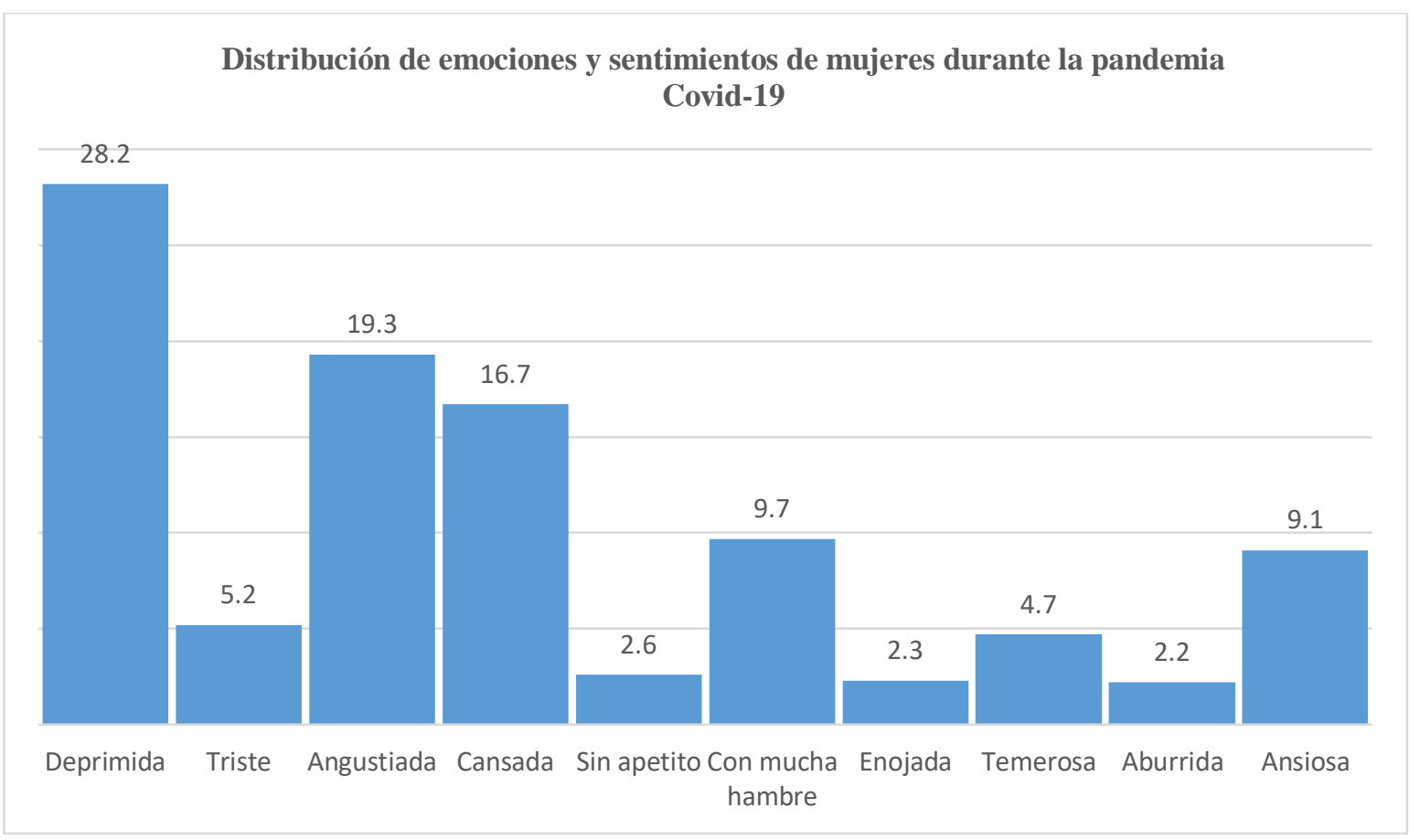

Nota: Elaboración propia con base en encuesta aplicada. 2020. El tiempo en tiempos del confinamiento.

En este sentido podemos observar que la incertidumbre social y el exceso de trabajo, que estamos viviendo como resultado del confinamiento provocado por la pandemia, alteró la dinámica de nuestras rutinas personales y provocó una gama de sentimientos y emociones en muchas mujeres; depresión angustia y miedo, como expresión de una realidad incierta que impacta en la salud y desencadena una visión negativa del futuro, y altera sus patrones de conducta y relaciones afectivas, estos resultados coinciden con una investigación realizada en España (Balluerka, N. et.al. 2020).

Yo estoy desesperada, angustiada, molesta, ya ni sé...al final ¿qué me queda? ¿Resignarme? Abrí mi negocio de regalos y ropa de niños pensando que podríamos tener en un ingreso extra y al final resultó siendo un gasto más, por la renta. Las clases de inglés que daba en la primaria que no era mucho, pero me daban cinco pesos por niño, la clase y tomaban dos clases de hora y media a la semana, cincuenta niños pues si eran buenos (mil pesos extra a la quincena). Con esto del encierro pues pasaron muchas cosas, se enferma mi mamá y tuvimos que atenderla con médico particular, un gasto. Se enferma mi suegro y fallece, luego el abuelo de mi esposo, que también fallece. Tener un enfermo en casa implica más gastos también y no me pesa porque es 


\section{Journal of the Academy | $22 \mid$}

mi mamá, pero mis hermanos no me ayudan. Tengo esto del quehacer, las clases en la tele de mis hijos, abrir un rato el local, me estoy enfermando del estómago a cada rato y no quiero ir al doctor para no gastar. (López, 2020).

Las emociones y los sentimientos son otra de las condiciones, cuyo impacto psicológico ha causado fuertes estragos en la vida cotidiana de las mujeres, un tanto por desafíos a los que se enfrentan, por los efectos de la pandemia y otro por experiencia del sobre trabajo que las obliga a buscar estrategias que les permita afrontar la difícil condición económica por la que muchas familias están pasando. Sin embargo, las mujeres buscan gobernar sus emociones y sentimientos, frente a sus familiares y replantar la visión de sus tareas como una acción gratificante y significativa, vinculada a valores personales.

De esta manera el sobre trabajo, la angustia, la depresión y el miedo, intervienen de manera directa en la autoestima de muchas mujeres que las hace vulnerables y quebranta su estado de ánimo, dificultándoles el desempeño de sus tareas y auto justificando su papel frente a los demás. Se trata digámoslo así, de un empobrecimiento en su autopercepción que les hace justificar la desigualdad en la responsabilidad de las tareas del hogar y la cosificación de su condición como mujer.

\section{Figura 4:}

Distribución de encuestados por dedicación

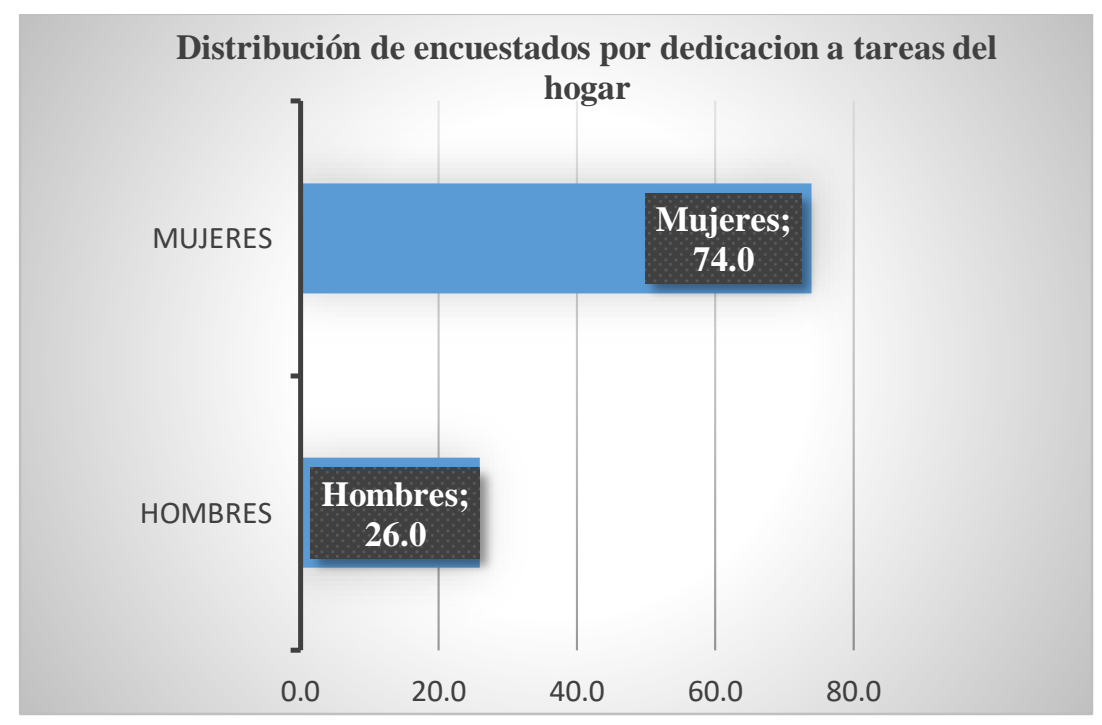

Nota: Elaboración propia con base en encuesta aplicada. 2020. El tiempo en tiempos del confinamiento. 


\section{Journal of the Academy $|23|$}

Las tareas realizadas por las mujeres dentro del hogar siguen siendo atribuidas casi de manera exclusiva a ellas, afectando su condición de salud, precarizando su fuerza de trabajo y el bienestar individual de y su familia, así estos meses de confinamiento no solo ha subrayado los pendientes en materia de equidad y desigualdad de género en la coparticipación de responsabilidades en tareas del hogar. La condición asimétrica del trabajo excesivo, que desplaza a un segundo plano la vida personal, como resultado de la doble triple jornada de trabajo.

De esta manera el prisma cotidiano de la vida de una mujer se sujeta a la dinámica del sobretrabajo y a las analogías de la sobre explotación en el hogar, sumadas al trabajo informal que ha venido realizando como resultado de la condición provocada por la pandemia. Es una situación normalizada por nuestra cultura que impone tareas vistas como parte de la reproducción humana y desde donde se puede observar un entramado de relaciones sociales que explican una condición de vulnerabilidad y situaciones que vuelven frágil a la mujer, por la violencia, marginación y explotación a la que es sometida culturalmente.

Si bien son condiciones impuestas culturalmente, hoy el fenómeno de la pandemia ha venido a señalar esa base de relaciones verticales del sobretrabajo en las mujeres, que predominan como un modo de socialización naturalizado y articulado subjetivamente a la idea del patrón jerárquico de la reproducción humana.

\section{CONCLUSIONES}

El panorama que muchos mexicanos hemos visto en estos meses de auto confinamientos como resultado de la pandemia, dibuja un escenario desalentador, sin precedentes para muchas familias, por su afectación económica y emocional. Se trata de una experiencia inédita que ha provocado insatisfacción, enojo y frustración. Muchas mujeres perdieron su empleo, otras más vieron reducidos sus ingresos, llevándolas a buscar estrategias para sobre vivir y con ello a vivir emociones y sentimientos negativos que ponen en riesgo su condición de salud y alteran su conducta social.

La condición laboral y de vulnerabilidad de este tiempo, ha roto la escasa sinergia del trabajo en el hogar, dificultando la empatía y la escasa estabilidad de las relaciones interpersonales en 


\section{Journal of the Academy | $24 \mid$}

las familias. Muchas mujeres ante la inestabilidad laboral han extendido su desempeño en otras tareas, a modo de obtener ingresos extras, a costa de un desequilibro en sus rutinas y una sobrecarga de trabajo, pero como hemos podido observar, el problema de la sobre carga de trabajo, ha desencantado emociones y experiencias cuyo aprendizaje, no solo impacto al ámbito de lo personal, ha sido también una experiencia colectiva que provoco disfunciones, afecto relaciones y reorganizo las convivencia en la familia.

Pero el problema central que hemos podido advertir, radica en uno de los efectos inmediatos del Covid-19: las mujeres y su impacto en el sobretrabajo, que por desgracia en su condición de fragilidad se encuentra sumada a la relación de su nivel económicas y pobreza, las cuales repercuten en sus estado físico y emocional de muchas mujeres por su condición social y cultural, tienen que padecer la sobrecarga de un trabajo invisible no reconocido, y que en nuestra cultura es visto como el costo, sacrificio y práctica correcta y esperada de una ama de casa.

\section{REFERENCIAS BIBLIOGRÁFICAS}

Balluerka, N. et.al. (2020). Las consecuencias psicológicas de la COVID 19 y el confinamiento. Servicio de publicaciones de la Universidad del País Vasco. España: Euska Herriko Unibertsitateko Argitalpen Zerbitzua. https://www.ub.edu/web/ub/ca/menu_eines/noticies/docs/Consecuencias_psicologicas_ COVID-19.pdf

Campillo, F. (2000). El trabajo doméstico no remunerado en la economía. Nómadas, 12, 98115.

http://nomadas.ucentral.edu.co/nomadas/pdf/nomadas_12/12_10C_Eltrabajodomestico noremunerado.PDF

Cisneros, J. (2010). 25 días de miedo. Periódico digital El Cotidiano, (159). https://biblat.unam.mx/hevila/ElCotidiano/2010/no159/6.pdf

Cisneros J. (2015). Visiones contemporáneas de la violencia. Universidad Autónoma Metropolitana. Unidad Xochimilco. México: Ediciones EÓN.

Delphos Nudge UNIT. (2020). Violencia contra las mujeres al alza en todo México. Recuperado de https://delphos.nu/violencia-contra-las-mujeres-al-alza-en-todo-mexico/

ENH. (2017). Encuesta Nacional de los Hogares. Instituto Nacional de Estadística, Geografía $e$ Informática. México. INEGI, GOB. Recuperado de https://www.inegi.org.mx/programas/enh/2017/

Hernández, E. (2006). El clientelismo en México: los usos políticos de la pobreza. Espacios Púbicos, 9(17). Universidad Autónoma de Estado de México, México. 


\section{Journal of the Academy $|25|$}

INEGI (2020). Encuesta sobre el Impacto económico generado por el COVID-19 en la actividad económica $y$ el mercado laboral. https://www.inegi.org.mx/programas/ecovidie/2020/

INEGI. (2020). El INEGI presenta resultados del impacto del COVID-19 en la actividad $\begin{array}{lllll}\text { económica } & y & \text { el mercado laboral. }\end{array}$ https://www.inegi.org.mx/app/saladeprensa/noticia.html?id=5828

Informe COVID-19. (2020). Las dos pandemias. Violencia contra las mujeres en México en contexto del COVID-19. México: Informe INTR-RNR EQUIS. https://equis.org.mx/wpcontent/uploads/2020/08/informe-dospandemiasmexico.pdf

Jaramillo, M. y Vásquez, D. (2018). La desigualdad global contenida en México. Nexos. Recuperado de https://economia.nexos.com.mx/?p=981

Lagarde, M. (1993). Los cautiverios de las mujeres: madresposas, monjas, putas, presas y locas. Universidad nacional Autónoma de México. Colección Posgrado, México.

Monroy, M. (2020a). En México, la brecha salarial de género es mayor en empleos de menores remuneraciones. Animal Político. Consultado el 18 de agosto de 2020. Recuperado de https://www.animalpolitico.com/simetria-datos-con-rostro/en-mexico-la-brechasalarial-de-genero-es-mayor-en-empleos-de-menores-remuneraciones/

Monroy, J. (2020b). Segob: violencia intrafamiliar aumentó 120\% desde la emergencia del Covid-19. Recuperado de https://www.eleconomista.com.mx/politica/Segob-violenciaintrafamiliar-aumento-120-desde-la-emergencia-del-Covid-19-20200416-0111.html

Reza, A. (2020). En enero de 2020, cada día 10 mujeres fueron asesinadas. Milenio Diario. https://www.milenio.com/policia/en-enero-de-2020-cada-dia-10-mujeres-fueronasesinadas 